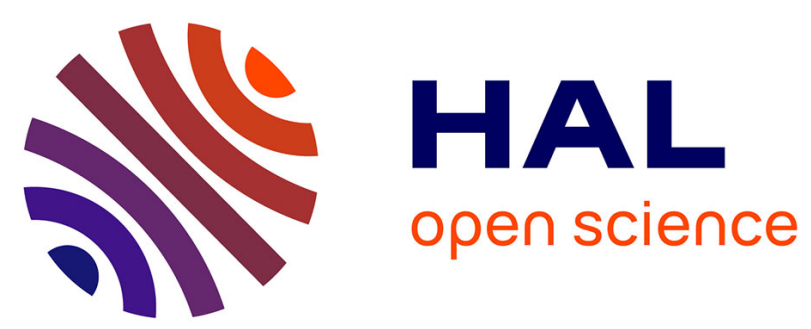

\title{
Rotational spectroscopy of imidazole: Accurate spectroscopic information for three vibrationally excited states and the heavy-atom isotopologues up to $295 \mathrm{GHz}$
}

B.E. Arenas, G. Batra, A.L. Steber, Luca Bizzocchi, A. Pietropolli Charmet, B.M. Giuliano, P. Caselli, B.J. Harris, B.H. Pate, Jean-Claude Guillemin, et al.

\section{To cite this version:}

B.E. Arenas, G. Batra, A.L. Steber, Luca Bizzocchi, A. Pietropolli Charmet, et al.. Rotational spectroscopy of imidazole: Accurate spectroscopic information for three vibrationally excited states and the heavy-atom isotopologues up to $295 \mathrm{GHz}$. Journal of Molecular Spectroscopy, 2021, 378, pp.111452. 10.1016/j.jms.2021.111452 . hal-03249672

\section{HAL Id: hal-03249672 \\ https://hal.science/hal-03249672}

Submitted on 11 Jun 2021

HAL is a multi-disciplinary open access archive for the deposit and dissemination of scientific research documents, whether they are published or not. The documents may come from teaching and research institutions in France or abroad, or from public or private research centers.
L'archive ouverte pluridisciplinaire HAL, est destinée au dépôt et à la diffusion de documents scientifiques de niveau recherche, publiés ou non, émanant des établissements d'enseignement et de recherche français ou étrangers, des laboratoires publics ou privés. 


\title{
Rotational spectroscopy of imidazole: Accurate spectroscopic information for three vibrationally excited states and the heavy-atom isotopologues up to 295 \\ GHz
}

\author{
Benjamin E. Arenas ${ }^{a, b, \#}$, Gayatri Batra ${ }^{a, b, \#}$, Amanda L. Steber ${ }^{a, b}$, Luca Bizzocchi ${ }^{c}$, \\ Andrea Pietropolli Charmet ${ }^{d}$, Barbara M. Giuliano ${ }^{c}$, Paola Caselli ${ }^{c}$, Brent J. Harris ${ }^{e}$, \\ Brooks H. Pate ${ }^{e}$, Jean-Claude Guillemin ${ }^{f}$, and Melanie Schnell ${ }^{a, b, *}$ \\ ${ }^{a}$ Deutsches Elektronen-Synchrotron (DESY), Notkestraße 85, 22607 Hamburg, \\ Germany \\ ${ }^{b}$ Institut für Physikalische Chemie, Christian-Albrechts-Universität zu Kiel, \\ Max-Eyth-Straße 1, 24118 Kiel, Germany \\ ${ }^{c}$ Center for Astrochemical Studies, Max-Planck-Institut für extraterrestrische Physik, \\ Gießenbachstraße 1, 85748 Garching, Germany \\ ${ }^{d}$ Dipartimento di Scienze Molecolari e Nanosistemi, Universit Ca' Foscari Venezia, \\ via Torino 155, 30172 Mestre, Italy \\ ${ }^{e}$ Department of Chemistry, University of Virginia, McCormick Road, Charlottesville, \\ VA 22903, USA \\ ${ }^{f}$ Univ Rennes, Ecole Nationale Suprieure de Chimie de Rennes, CNRS, IRCRUMR \\ 6226, F-35000 Rennes, France \\ ${ }^{\#}$ These authors are co-first authors of the work. \\ *Corresponding author, e-mail: melanie.schnell@desy.de.
}

Submitted December 22, 2020. Revised April 10, 2021.

\begin{abstract}
We report our analysis of the pure rotational spectra of low-lying vibrationally excited states and heavy-atom rare isotopologues of imidazole. To facilitate searches for imidazole in the interstellar medium, we previously described the analysis of the rotational spectrum of the imidazole main isotopologue across the 2-295 GHz range, and we extend this analysis here. Structure optimisation and anharmonic frequency calculations were performed to aid the spectral analysis. Three vibrationally excited states of imidazole were assigned in our room-temperature spectra, with energies up to approximately $670 \mathrm{~cm}^{-1}$ above the vibronic ground state. The vibrational states could act as temperature probes in warmer star-forming regions. The ${ }^{13} \mathrm{C}$ and ${ }^{15} \mathrm{~N}$ heavy-atom isotopologues were assigned, which allowed for the structure of imidazole in the gas phase to be determined. Structural comparisons are drawn between the related heterocyclic molecules hydantoin and imidazolidine. An experimental gas-phase structure of the former is also determined in this work. The potential detection of the isotopologues could help deduce formation pathways towards imidazole and other nitrogencontaining cyclic compounds in interstellar space.
\end{abstract}




\section{Keywords}

astrochemistry, imidazole, isotopologues, molecular structure, rotational spectroscopy, vibrationally excited states

\section{Highlights}

- Imidazole was studied between 2-295 GHz; hydantoin was studied between 18 $26 \mathrm{GHz}$.

- Three vibrationally excited states of imidazole were observed and assigned.

- Isotopologue analysis allowed gas-phase structure determination for both molecules.

- Imidazole, hydantoin, and imidazolidine structures and vibrations are compared.

- Precise rotational constants and line lists are delivered for all assigned species.

\section{Graphical Abstract}

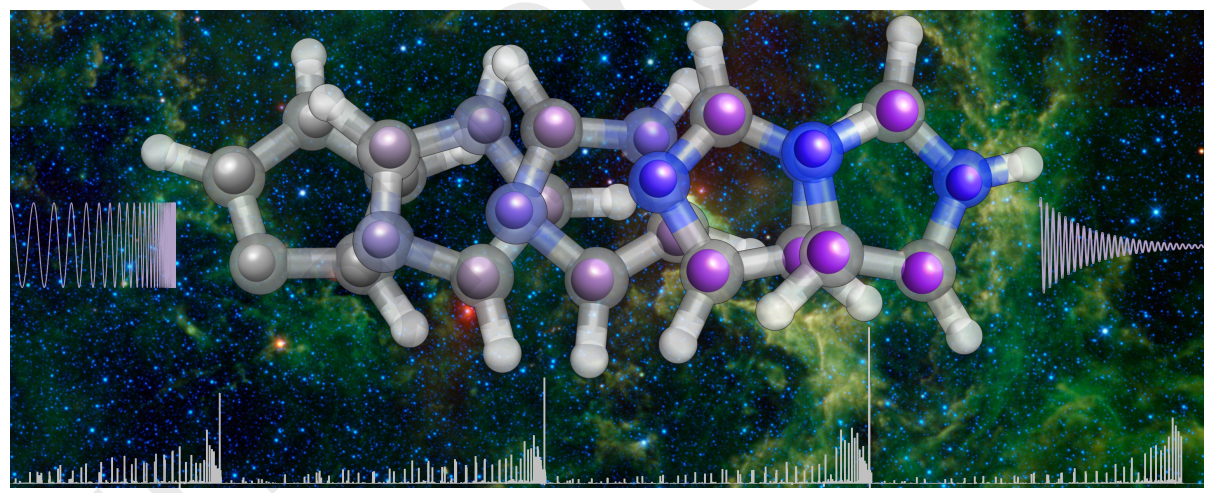




\section{Introduction}

Activities to fully characterise the chemical inventory of the interstellar medium (ISM) are key drivers for laboratory astrochemistry, with over 200 molecules having been identified in the ISM so far [1]; an up-to-date list can be found at the Cologne Database for Molecular Spectroscopy ${ }^{1}$. In particular, the increase in sensitivity of observational facilities such as the Atacama Large Millimeter/submillimeter Array (ALMA) is facilitating the detection of more complex molecules. The availability of accurate rest frequencies and precise rotational constants for astrochemically relevant molecules, such as complex organic molecules (COMs), must follow these advancements in observational astronomy. These experimentally determined data sets are necessary for searches for these molecules in the ISM, and we present here such data that will allow searches for vibrationally excited states and isotopologues of imidazole $\left(c-\mathrm{NHCHN}(\mathrm{CH})_{2}\right)$ to be performed in observational spectra.

Imidazole is an interesting molecule for astrochemical studies due to the confirmed presence of N-heterocycles on meteorites (see Ref. [2] and references therein), suggesting conditions exist in space for its formation in substantial abundance. N-heterocycles are also prevalent in biologically important motifs. For example, imidazole is present in the purine bases of DNA and RNA, as well as in the structures of the amino acid histidine and the neurotransmitter histamine. Despite a number of cyclic compounds already being observed in the ISM, imidazole has yet to be detected. Indeed, following on from an unsuccessful search for imidazole by the authors of Ref. [3], our analysis of the ground state rotational spectrum was accompanied with an observational search towards the molecular core Sagittarius B2(N2) with the EMoCA line survey [4], which resulted in a non-detection [5]. We had also previously suggested a new search towards TMC-1, where benzonitrile was detected in 2018 [6]. The presence of 1-cyano-1,3-cyclopentadiene [7] and 1- and 2-cyanonaphthalene [8] towards the same source was recently reported. These detections, as concluded in Ref. [7], hint towards a reservoir of ring molecules in TMC-1 that defies the current chemical models, and this could prove promising in the search for imidazole.

Vibrationally excited states and isotopologues are important considerations when analysing observational data sets [9]. Indeed, as noted in Ref. [10], these species are believed to be the source of many unidentified lines. The percentage of unidentified lines can be as much as 70\% in very deep observations at ALMA Bands 3 (84-116 $\mathrm{GHz})$ and $6(211-275 \mathrm{GHz})$ [11]. Further, the 2018 NASA Laboratory Astrophysics Workshop identified "spectral studies producing rotational line lists, especially focusing on vibrational states, [and] isotopologues as one of the highest-priority recommendations [12]. The experimentally determined rotational constants and line lists, like those we report here, facilitate searches for these species in the ISM.

Vibrationally excited states are important as indicators of the physical conditions of the regions they are detected in (see, for example, Ref. [13] for a comparison between vibrational and local thermal equilibrium temperatures), and they can thus aid in the characterisation of, for example, hot core regions. The lines generated by vibrational states of already-detected molecules can also be considered as 'weeds' [14] or a hindrance to identifying new COMs in observational data sets. Their elimination from

\footnotetext{
${ }^{1}$ ht tps://cdms. astro.uni-koeln.de/cdms/portal/
} 
astronomical spectra is an important step in processing complex, confusion-limited spectra. Knowledge of these lines can draw attention to cases of blended transitions and prevent misassignments, particularly in broadband, unbiased observational campaigns.

From an astrochemical perspective, the search for and detection of the ${ }^{13} \mathrm{C}$ and ${ }^{15} \mathrm{~N}$ isotopologues in regions where imidazole is detected could be of importance for elucidating formation pathways of the parent species. Furuya et al. demonstrated with a gas-grain chemical network model that isotopic ratios can be used to deduce likely routes to interstellar molecules. In their study, they showed that the ${ }^{12} \mathrm{C} /{ }^{13} \mathrm{C}$ ratio for $\mathrm{CCH}$ and $\mathrm{CCS}$ differed from the elemental ${ }^{12} \mathrm{C} /{ }^{13} \mathrm{C}$ ratio, and the ratio was dependent on whether the molecule was formed from the carbon ion or from carbon monoxide [15]. One could postulate that constructing similar models with the nitrogen atom/ion or other small nitrogen-containing species (some examples are discussed below) could allow for the deduction of likely formation pathways for imidazole. Any future detection of imidazole isotopologues, made possible by the high-level line lists provided in this study, would provide the interstellar abundances and ${ }^{14} \mathrm{~N} /{ }^{15} \mathrm{~N}$ ratios required for such an analysis to be performed.

Isotopologues are also important species from a spectroscopic and structural point of view. With the experimentally determined rotational constants of the singly-substituted isotopologues, a substitution structure using Kraitchman's equations can be determined [16]. Blackman et al. presented an analysis of the rotational spectrum of imidazole and $\mathrm{N}$-deutero imidazole, which focussed on the characterisation of the quadrupole hyperfine structure [17]. Building on this, and their previous study in Ref. [18], Christen et al. provided a set of rotational constants for the molecule's parent species, as well as the $\mathrm{D},{ }^{13} \mathrm{C}$, and ${ }^{15} \mathrm{~N}$ isotopologues determined from data up to $40 \mathrm{GHz}$ [19]. This allowed a comprehensive structure of imidazole in the gas phase to be constructed, which is re-evaluated here based on our extended data set.

Imidazole was previously studied with an astrochemical motivation by Schwell et al. [20], where the authors studied the fragmentation patterns of imidazole with photoionisation mass spectrometry. The survival of imidazole, and the other nitrogencontaining cyclic molecules studied, was discussed. In summary, the authors of Ref. [20] conclude that protection from vacuum ultraviolet (VUV) radiation is required for detectable quantities of the studied N-bearing molecules to survive in the ISM. The molecules would likely undergo dissociative ionisation if irradiated with VUV radiation. It was therefore suggested that searches for imidazole be done in hot molecular cores and in young circumstellar disks, where such shielded conditions are likely to exist. If a search for the imidazole ground state in such an environment would be successful, knowledge of the rotational parameters for vibrational states would be important for further searches.

A number of previous studies have looked at the incorporation of smaller molecules, such as HCN, into larger ring structures. For example, acetylene polymerisation with $\mathrm{HCN}$ in the gas-phase has been found to produce nitrogen-containing polycyclic aromatic structures [21, 22, 23], which could have interesting implications for interstellar imidazole synthesis. A further example of this was included in Ref. [24], where a number of imidazole-like structures were presented in an interstellar synthetic pathway towards adenine. The pathways start from familiar small molecules - HCN, HCCN, 
$\mathrm{NH}_{2} \mathrm{CN}$, and $\mathrm{CN}$ - and one could imagine termination of the reaction at any of the relevant steps could result in imidazole being formed.

In this paper, we present the analysis of the rotational spectra of the imidazole vibrationally excited states and heavy-atom isotopologues up to $295 \mathrm{GHz}$. The resulting rotational constants, quadrupole coupling constants, and high order distortion constants have allowed us to accurately predict the rotational line lists for these species in the millimeter-wave regime and beyond. This data is essential for observational searches. Further, the motions of the assigned vibrational states were revealed from the results of anharmonic frequency calculations. The isotopologue analysis allowed us to characterise the gas-phase structure of imidazole's ring motif and compare its structural parameters to related molecules, including hydantoin $\left(c-\mathrm{NHC}(\mathrm{O}) \mathrm{NHCH}_{2} \mathrm{C}(\mathrm{O})\right)$, whose heavy-atom isotopologues were also experimentally examined in this work.

\section{Experimental Details and Theoretical Methods}

The experimental work was conducted across a number of high-resolution broadband chirped-pulse Fourier transform rotational spectrometers at the Deutsches ElektronenSynchrotron (DESY, Germany) and at the University of Virginia (USA). The work covers the frequency ranges $2-8,12-15.5,18-26,75-110$, and 260-295 GHz. The experiments reported on here were performed for the analysis of the imidazole main isotopologue as presented in Ref. [5], where there was no analysis presented on the vibrationally excited states nor the isotopologues. The methodology has therefore been described in detail previously, and a short summary follows here for information.

The Hamburg COMPACT spectrometer was used to record the spectrum of imidazole across the $2-8 \mathrm{GHz}$ and $12-15.5 \mathrm{GHz}$ regions [25, 26, 27]. For these measurements, solid imidazole (purchased from Sigma Aldrich, $\geq 99 \%$ purity, and used without further treatment) was placed in a reservoir nozzle, which was heated to $90^{\circ} \mathrm{C}$. Neon, at a pressure of 3 bar, was used as a backing gas, and the imidazole vapour was supersonically expanded into the vacuum chamber. The molecules then interacted with the incident radiation, which is created by an arbitrary waveform generator (AWG) and amplified by a $300 \mathrm{~W}$ travelling wavetube amplifier (for the $2-8 \mathrm{GHz}$ experiment) or a 50 W solid state amplifier (for the $12-15.5 \mathrm{GHz}$ experiment) before being transmitted into the chamber. The spectrometer uses the fast frame method, whereby each molecular pulse is probed by eight chirps spanning the frequency regime of the experiment. The relaxation of the induced macroscopic dipole moment is collected in the form of a free induction decay (FID). For the 2-8 GHz experiment, 1.6 million FIDs (each recorded for $40 \mu \mathrm{s}$ ) were co-added and fast Fourier transformed into the frequency domain. For the 12-15.5 GHz experiment, 1.2 million FIDs (each recorded for $20 \mu \mathrm{s}$ ) were co-added and fast Fourier transformed.

The spectrum across the $18-26 \mathrm{GHz}$ region was recorded using a segmented chirpedpulse Fourier transform microwave spectrometer [27]. The spectrometer combines the segmented approach and the multi-train method. Here, the segmented approach involves splitting the spectrum into $800 \mathrm{MHz}$ segments, and the spectrum across the whole bandwidth is obtained by concatenating the segments together [28]. The multitrain method involves utilising multiple pulse trains per molecular pulse [27], which is similar to the fast frame approach exploited in the Hamburg COMPACT spectrometer. The $1.5 \mu$ s excitation pulses are written by an AWG, and they cover the region 
7-3 GHz. The pulses are then frequency up-converted with a number of mixing and doubling stages. The FIDs were collected, frequency down-converted, then recorded for $10 \mu \mathrm{s}$. Segment concatenation yielded the spectrum across the whole region, which consisted of 1.8 million co-added FIDs. Full details of the design and characterisation of the instrument are given in Ref. [27]. The imidazole sample was placed in a heatable reservoir nozzle similar to that used in the experiments performed on the COMPACT. Three bar backing pressure of neon was used as the carrier gas. The rotational spectrum of hydantoin (also purchased from Sigma Aldrich, $>99 \%$ purity, and used without further treatment) was also recorded in the $18-26 \mathrm{GHz}$ region. This was performed in extension to the work in Ref. [29] and in order to obtain an experimental gas-phase structure that could be used for structural comparisons between imidazole and hydantoin (see Section 3). For the hydantoin experiment, the sample was placed in an internal reservoir nozzle, heated to $185^{\circ} \mathrm{C}$, and supersonically expanded into the vacuum chamber with 3 bar of neon as the backing gas. The spectrum consists of 3.5 million co-added FIDs. The frequency accuracy achieved in both the COMPACT and the $18-26 \mathrm{GHz}$ spectrometer was in the order of $25 \mathrm{kHz}$.

The spectrum in the $75-110 \mathrm{GHz}$ region was recorded in a room temperature flow cell set-up with a BrightSpec, Inc. W-band spectrometer. This spectrometer also utilises the segmented approach, and the $35 \mathrm{GHz}$ of bandwidth is covered in segments of $30 \mathrm{MHz}$, as implemented in the instrument's High Dynamic Range (HDR) mode [30]. The excitation pulses are created by an AWG in the $1.5-2.3 \mathrm{GHz}$ range, and the millimeter-wave radiation is achieved with an up-conversion stage and an active multiplier chain (AMC, x6). The excitation pulses were $500 \mathrm{~ns}$ in duration, and after collection and frequency down-conversion, the FIDs were recorded for $4 \mu \mathrm{s}$. A total of 500,000 FIDs were co-added in the W-band experiment. The imidazole sample was heated to $90{ }^{\circ} \mathrm{C}$ in an external reservoir, and the vapours were pulsed into the sample chamber without a backing gas.

The $260-295 \mathrm{GHz}$ spectrum was recorded at the University of Virginia. The instrument has been previously described in detail [28, 31, 32]. The operation of the 260-295 $\mathrm{GHz}$ spectrometer also follows the segmented operating regime. The AWG produces a pulse train in the region 2-4 GHz, which is then frequency up-converted by mixing and an AMC to the $260-295 \mathrm{GHz}$ range. The spectrum is split into fifty segments of 720 MHz bandwidth, where each $720 \mathrm{MHz}$ is made up of thirty $24 \mathrm{MHz}$ chirps in a similar fashion to the HDR mode of the W-band spectrometer. The pulse duration was $100 \mathrm{~ns}$, and the FIDs, after collection and frequency down-conversion, were recorded for 1.9 $\mu \mathrm{s}$. The experiment was performed with imidazole crystals, which were sublimed, and the vapours were allowed to enter the sample chamber, where a pressure of $7 \mu$ bar was maintained in a slow-flow configuration. The number of co-added FIDs in this case was 10,000 , which equates to approximately three minutes of measurement time. The frequency accuracy achieved in both the W-band spectrometer and the $260-295 \mathrm{GHz}$ spectrometer was about $35 \mathrm{kHz}$.

The assignment of the imidazole parent species was presented and discussed in our previous work [5]. Using the same experimental data, we were able to assign the rotational spectra of the singly-substituted heavy isotopologues in natural abundance. This was achieved by combining and refining the accurately determined nitrogen $\left({ }^{14} \mathrm{~N}\right)$ quadrupole coupling constants and high order centrifugal distortion constants from Ref. 
[5] with the $A, B$, and $C$ rotational constants determined in Ref. [19]. A global fit was performed for the individual isotopologues, which involved fitting the rotational constants and the centrifugal distortion constants to the assigned line frequencies across all of the frequency regimes studied in this work. Further, the nuclear quadrupole coupling constants were fit only to transitions which exhibited hyperfine splitting (HFS) - in this work, this included the spectra up to $26 \mathrm{GHz}$. This global fit procedure was also used in the analysis of the rotational spectrum of the parent imidazole isotopologue [5].

The spectra were initially fit using the PGOPHER programme [33]. Global fits were obtained using Pickett's SPFIT/SPCAT suite of programmes [34]. The data were fit to Watson's $S$-reduction rotational Hamiltonian in the III $^{l}$ representation with centrifugal distortion corrections and hyperfine splitting considerations. The substitution structure analysis was performed using the KRA programme [35], and the EVAL programme [35] was used to determine structural parameters and their associated errors; the effective structure analysis was performed using the STRFIT programme [36].

Extensive ab initio calculations have been performed to derive theoretical predictions for many spectroscopic and structural parameters of imidazole and its heavy-atom isotopic variants. The computational treatment included complete basis set extrapolation (CBS) by using several calculations carried out at both the Hartree-Fock and the $\mathrm{CCSD}(\mathrm{T})$ levels of theory in conjunction with basis sets up to cc-pV6Z and a composite approach for modelling core-valence and full electron correlation contributions. Anharmonicity corrections to the vibrational fundamentals and to vibrationally-averaged rotational constants have been computed at the MP2/cc-pVQZ level (hybrid approach). With regards to the calculation of the sextic centrifugal distortion constants, the fcCCSD level of theory in conjunction with the cc-pVTZ basis set has been chosen due to its good balance between accuracy and computational time (see, for example, Ref [37]) with respect to the more demanding fc-CCSD(T). All of these calculations have been carried out using the CFOUR suite of programmes ${ }^{2}$. Full details can be found in the preceding publication and the references therein [5].

Structure optimisation calculations were performed at the MP2/aug-cc-pVTZ level of theory as implemented in Gaussian09 [38] for hydantoin and imidazolidine. The hydantoin calculations supported the assignment of the isotopologues and the subsequent gas-phase structure determination. The imidazolidine calculations supplied theoretical parameters for the structural comparisons discussed later.

\section{Results and Discussions}

\subsection{Vibrationally Excited States}

The experiments in the $75-110 \mathrm{GHz}$ and $260-295 \mathrm{GHz}$ ranges were performed in room temperature flow cells, and thus the rotational spectra of low-lying vibrationally excited states could be observed and assigned. We present here the assignment of three of these vibrationally excited states: $v_{21}, v_{20}$, and $v_{19}$. The assignment process was supported by

\footnotetext{
${ }^{2}$ CFOUR, Coupled-Cluster techniques for Computational Chemistry, a quantum-chemical programme package written by J.F. Stanton, J. Gauss, L. Cheng, M.E. Harding, D.A. Matthews, P.G. Szalay et al., and the integral packages MOLECULE (J. Almlof and P.R. Taylor), PROPS (P.R. Taylor), ABACUS (T. Helgaker, H.J. Aa.Jensen, P. Jrgensen, and J. Olsen), and ECP routines by A. V.Mitin and C. van Wullen. For the current version, see http://www.cfour.de.
} 
theoretical calculations of the corresponding fundamental anharmonic frequencies and vibrational contributions to the rotational constants (see Section 2). The experimentally determined rotational constants are detailed in Table 1 . We observe a very good agreement between the experimental and theoretical rotational constants. It should be noted that the number of lines assigned per state varies, mainly due to the decrease in signal-to-noise ratio (SNR) with increasing vibrational state energy, which means that some weaker lines observable for lower energy states blend into the noise for higher energy states. The sextic centrifugal distortion constants were required, as was the case for the vibronic ground state [5] and isotopologues (see below), to obtain the best possible fit.

The motions associated with the assigned fundamental modes could also be inferred from anharmonic frequency calculations. The $v_{21}$ vibration is a stretch of the $\mathrm{N}(1)-\mathrm{H}$ bond, resulting in an out-of-plane displacement of the hydrogen atom. The $v_{20}$ and $v_{19}$ vibrations are wagging motions along the $\mathrm{C}(4)-\mathrm{C}(5)-\mathrm{N}(1)$ and the $\mathrm{N}(1)-\mathrm{C}(2)-\mathrm{N}(3)$ sections of the ring, respectively, which manifest as the out-of-plane displacement of the hydrogen atoms connected to these heavy atoms. Table 1 also contains depictions of these fundamental modes. Parts of the experimental spectra are shown in Figure 1 , and they are compared to the simulated spectra based on the fitted rotational constants for the assigned vibrational states. The spectra of these states, like those of the imidazole vibronic ground state and isotopologues in the same frequency region, are characterised by an intense Q-branch $(\Delta J=0)$ ladder, which culminates with a set of R-branch $(\Delta J=+1)$ transitions [5].

The rigidity of the imidazole ring motif may be hinted towards by the relatively high energy of the vibrationally excited states. The lowest energy vibrational state, $v_{21}$, has $\Delta \mathrm{E}^{e x p}=513 \mathrm{~cm}^{-1}$ relative to the imidazole ground state [39]. In contrast, imidazolidine's lowest energy vibrational state, $v_{33}$, is only $\Delta \mathrm{E}^{a}=71 \mathrm{~cm}^{-1}$ above the ground state at MP2/aug-cc-pVTZ, pointing towards the increased flexibility of the fully saturated structure.

The vibrationally excited states of a number of other aromatic molecules have been characterised, and one can gain valuable information from comparisons between such systems. The energies of the vibrationally excited states of the nitrogen-containing aromatic molecule indole have previously been reported. The energies of the $v_{42}$ and $v_{41}$ fundamental modes were determined as 207 and $241 \mathrm{~cm}^{-1}$, respectively [40, 41], and the rotational spectra of these states were analysed recently in Ref. [42]. The $v_{42}$ state is an out-of-plane butterfly motion, which deforms the ring structure [40]. These states are substantially lower in energy compared to the lowest vibrational states of imidazole. The first indole vibrational motion analogous to an imidazole motion is found about $740 \mathrm{~cm}^{-1}$ above the ground state, which is a C-H bending motion [43].

An important implication is the effect that the vibrational energies of imidazole may have on the probabilities of astronomical detection. The difference in the SNR between the experimental spectra of the imidazole ground state and the lowest energy vibrationally excited state, $v_{21}$, is a factor of 10 at approximately $300 \mathrm{~K}$. This factor matches well with the assumed Boltzmann distribution at $300 \mathrm{~K}$, which suggests a population difference between the vibronic ground state and the $v_{21}$ state of a factor of twelve. For hydantoin, a structurally related molecule to imidazole, for which a structural comparison is laid out in the next section, the ratio of the SNR of the ground state 
to the first vibrationally excited state $\left(140 \mathrm{~cm}^{-1}\right.$ above the ground state) was found to be 1:0.6 [44]. This intensity ratio was reported for the experiment at $420 \mathrm{~K}$ [44], and the Boltzmann population difference at that temperature is a factor of 1.5. This reinforces experimentally the higher relative energies of the imidazole vibrationally excited states. The detection of the imidazole vibrational states will therefore require searches in regions with relatively higher temperatures, where the states are more substantially populated. This can also be seen in the Boltzmann distributions at the lower temperatures. For example, in the Sagittarius B2 region N1S, where a rotational temperature of approximately $160 \mathrm{~K}$ has been previously determined [45], the ground state to $v_{21}$ state population difference is a ratio of 100:1. The Boltzmann population of the vibrationally excited states is virtually zero at the low temperatures of TMC- 1 . 


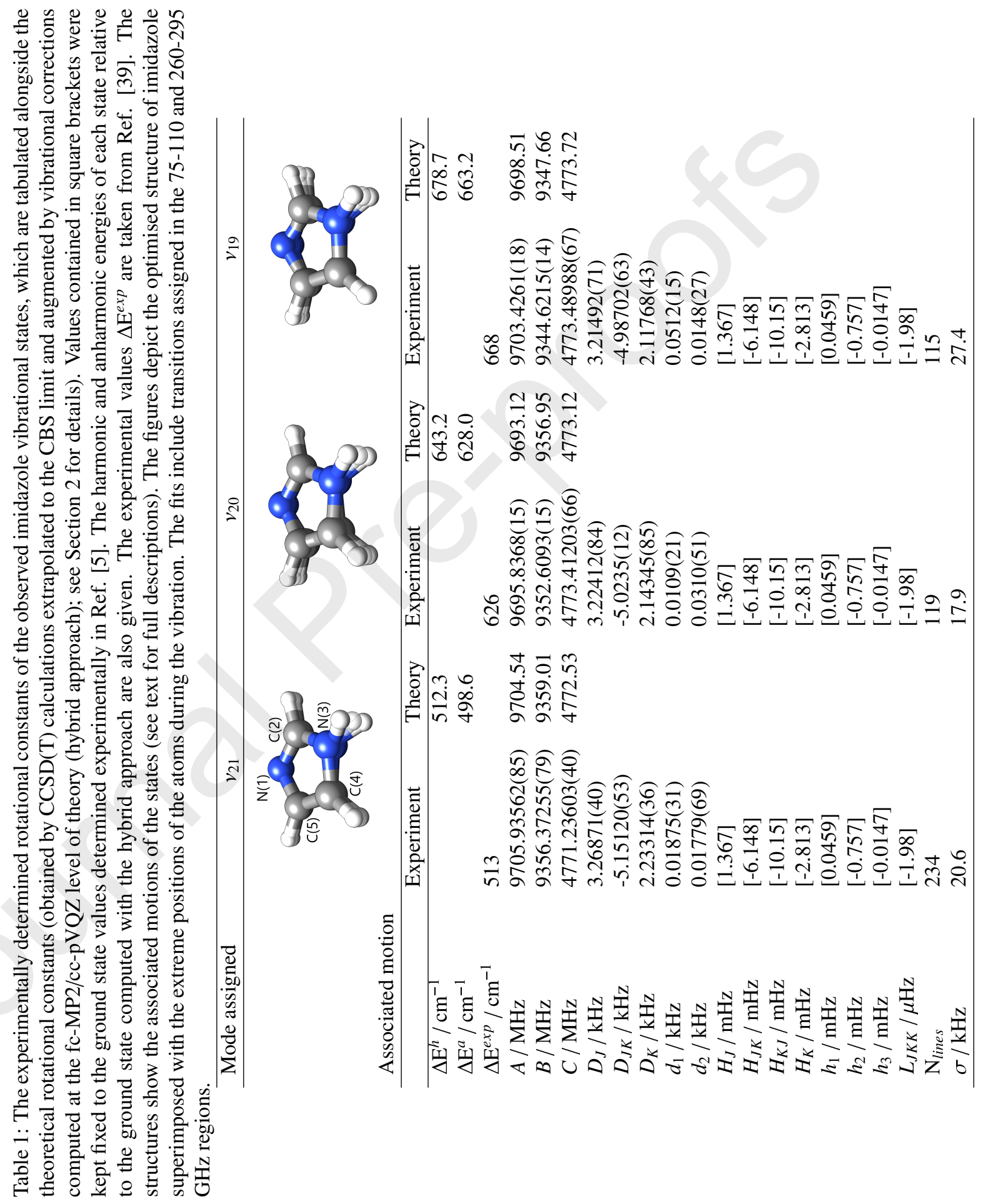



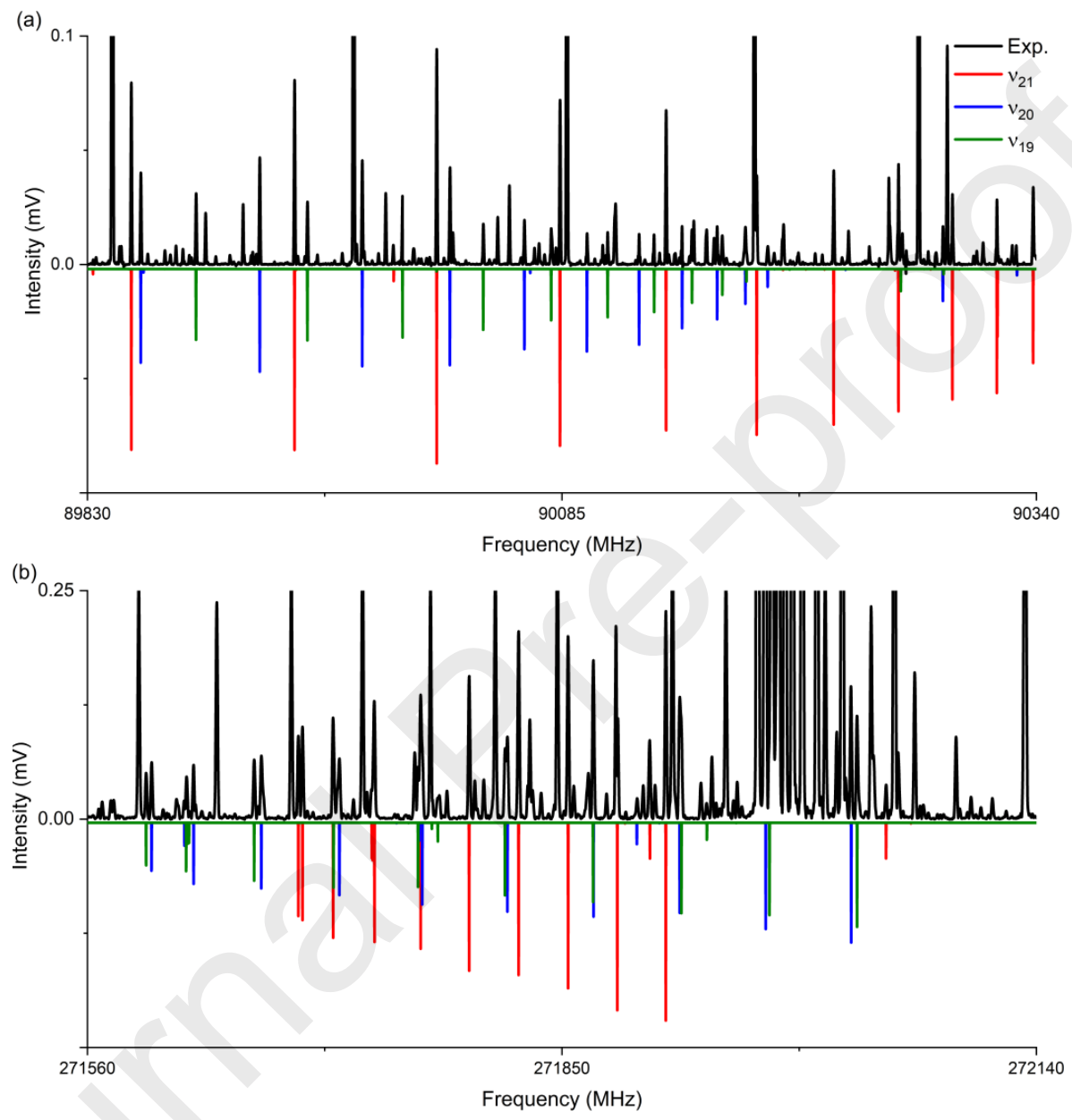

Figure 1: Parts of the rotational spectrum of imidazole (black) with the simulated spectra based on the experimentally determined rotational constants for the three assigned vibrationally excited states at $300 \mathrm{~K}\left(v_{21}\right.$ is shown in red, $v_{20}$ is shown in blue, and $v_{19}$ is shown in green). (a) A zoom-in of the $\mathrm{W}$-band spectrum, showing a number of assigned Q-branch transitions. (b) Assigned R-branch transitions of the normal modes in the 260-295 GHz region. The simulated spectra intensities have been normalised to the experimental spectrum for clarity. 


\subsection{Heavy-atom Isotopologues}

Experimental values for the ground state rotational constants of many isotopic variants of imidazole (three ${ }^{13} \mathrm{Cs}$, two ${ }^{15} \mathrm{Ns}$, four Ds, and one $1,2-\mathrm{D}_{2}$ ) were already available in the literature [19]. In addition to these rotational constants, we used theoretically computed values for the quartic and sextic distortion constants (see Section 2) as the initial input for our spectral analyses. The spectral assignments of the singly-substituted ${ }^{13} \mathrm{C}$ and ${ }^{15} \mathrm{~N}$-isotopologues were thus accomplished in a straightforward way. We noted that, up to moderately low $J$ levels $(J \leq 25)$, the centrifugal distortion effects are small enough to be considered almost isotopically invariant, and the set of parameters determined for the parent species works equally well in modelling the rotational energy trends. As the value of $J$ increased $(25 \leq J \leq 50$ ), the use of isotopologuespecific centrifugal distortion constants becomes critical to achieve a satisfactory fit of the measured transition frequencies. The small $d_{1}$ and $d_{2}$ constants, in particular, display significant changes in magnitude among isotopologues. For the ${ }^{13} \mathrm{C}(2)$ and ${ }^{13} \mathrm{C}(5)$ isotopologues, the sign also changed with respect to the parent isotopologue's distortion constants. While this effect is predicted by the ab initio results (see Table 2), it is a less commonly observed phenomenon upon isotopic substitution of a heavy atom, and the behaviour is worth a more detailed treatment. The distortion constants are a linear combination of the values $\tau_{\alpha \beta \gamma \delta}^{\prime}$, which are introduced as an energy correction term in the Hamiltonian of the rigid rotor to account for distortion [46]. The six $\tau_{\alpha \beta \gamma \delta}^{\prime}$ values relevant for the determination of the quartic centrifugal distortion constants are $\tau_{\text {aaaa }}^{\prime}$, $\tau_{b b b b}^{\prime}, \tau_{c c c c}^{\prime}, \tau_{a a b b}^{\prime}, \tau_{a a c c}^{\prime}$, and $\tau_{b b c c}^{\prime}$. The underlying theory and relevant equations used to determine these values can be found in Ref. [46].

The calculated $\tau_{\alpha \beta \gamma \delta}^{\prime}$ for all the heavy-atom isotopologues (see Table 2) do not show significant deflection from the values obtained for the parent isotopologue. These $\tau_{\alpha \beta \gamma \delta}^{\prime}$ values can be linearly combined to give the quartic centrifugal distortion constants. Upon isotopic substitution, the inertial axes can shift, which can result in a small change in the moment of inertia elements in the inverse moment of inertia matrix. This can lead to a small change in the $\tau_{\alpha \beta \gamma \delta}^{\prime}$ values. Since the distortion constants are the linear combination of these $\tau_{\alpha \beta \gamma \delta}^{\prime}$ values, a change in magnitude and sign of the quartic centrifugal distortion constants may be possible, as is the case for imidazole, specifically seen in $d_{1}$ and $d_{2}$. The Supplementary Material contains a comparison between the values of $\tau_{a a a a}^{\prime}$ and $\tau_{b b b b}^{\prime}$ determined in this work and those determined in Ref. [19]. The analysis performed in this work increases the precision of these values by two orders of magnitude. The phenomenon of a change of magnitude or sign of the quartic centrifugal distortion constants upon isotopic substitution is typically more pronounced in lighter molecules such as water, $\mathrm{H}_{2} \mathrm{O}$, and hydrogen sulphide, $\mathrm{H}_{2} \mathrm{~S}$ [46], and ring structures, such as 1,3,4-oxadiazoles, $\mathrm{C}_{2} \mathrm{H}_{2} \mathrm{~N}_{2} \mathrm{O}$ [47], and pyridine, $\mathrm{C}_{5} \mathrm{H}_{5} \mathrm{~N}$ [48]. 


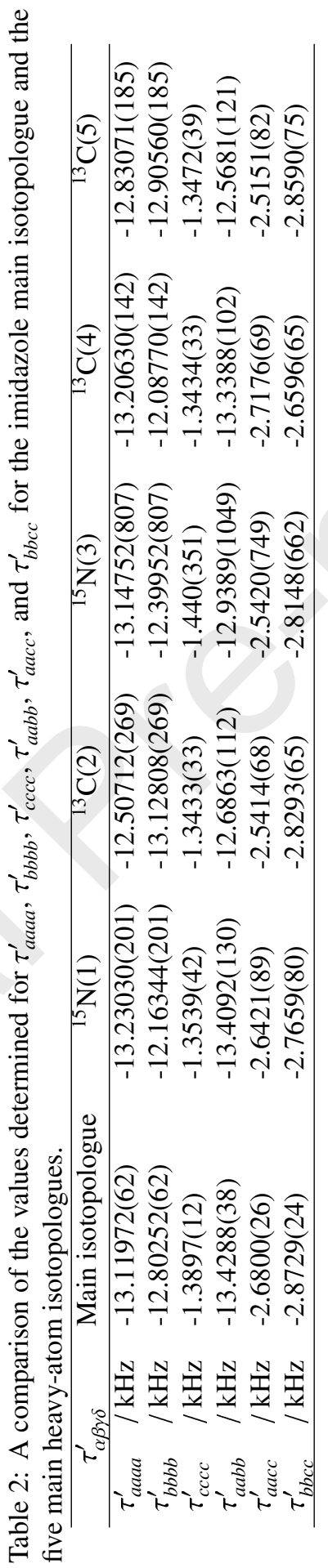


Table 3 shows the experimentally determined constants and compares them to those obtained theoretically. Figures 2 and 3 show the experimental spectrum compared with the simulated spectra of the fitted rotational constants for the assigned isotopologues. Note that Figure 2 shows a section of the low frequency data set (12-15.5 $\mathrm{GHz}$ ), where the spectra of the isotopologues exhibit HFS due to the presence of the ${ }^{14} \mathrm{~N}$ nucleus/nuclei. Figure 3 shows a section of the high frequency data set (260-295 $\mathrm{GHz}$ ), where this splitting is no longer present due to the collapse at the relatively high principal rotational energy level, $J$ (transitions assigned in the $260-295 \mathrm{GHz}$ region have $J$ values $17 \leq J \leq 52$ ). The parameters provided in Table 3 are a result of fitting the rotational constants and centrifugal distortion constants to all the assigned line frequencies and fitting quadrupole coupling constants only to line frequencies in the 2 $26 \mathrm{GHz}$ region, as described in the previous section.
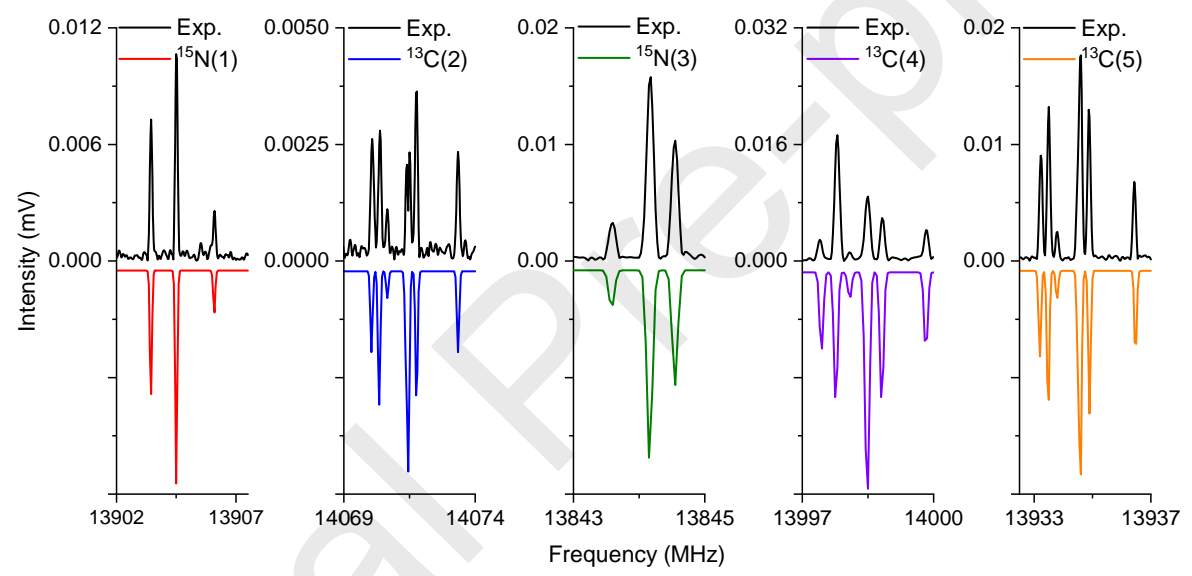

Figure 2: A zoom-in of the assigned spectra of the singly-substituted isotopologues of imidazole in the frequency range $12-15.5 \mathrm{GHz}$, where the $1_{0,1}-0_{0,0}$ transitions show HFS. The experimental spectra are shown in black, and the simulated spectra of the fitted rotational constants at $3 \mathrm{~K}$ are shown in colour. The atom labelling scheme is detailed in Table 1 and Figure 4. The simulated spectra intensities have been normalised to the experimental spectrum for clarity. 
Table 3: A comparison of the experimental rotational constants for the singlysubstituted heavy-atom isotopologues of imidazole determined in this work and those theoretically calculated. The theoretical rotational constants were obtained by $\operatorname{CCSD}(\mathrm{T})$ calculations extrapolated to the CBS limit and augmented by vibrational corrections computed at the fc-MP2/cc-pVQZ level of theory, the quartic centrifugal distortion constants were calculated at the fc-CCSD(T)/cc-pVQZ level of theory, and the sextic centrifugal distortion constants were calculated at the fc-CCSD/cc-pVTZ level of theory; see Section 2. The values contained in square brackets were kept fixed to theoretical values. Note that the value of $L_{J K K}$ was fixed to the value obtained from the main isotopologue analysis in Ref. [5]. The fits from this work include transitions assigned in the 2-8, 12-15.5, 18-26, 75-110, and 260-295 GHz regions. The positions of each isotopic substitution can be found in Table 1 and Figure 4.

\begin{tabular}{|c|c|c|c|c|c|c|}
\hline & \multicolumn{2}{|c|}{${ }^{15} \mathrm{~N}(1)$} & \multicolumn{2}{|c|}{${ }^{13} \mathrm{C}(2)$} & \multicolumn{2}{|c|}{${ }^{15} \mathrm{~N}(3)$} \\
\hline & Experiment & Theory & Experiment & Theory & Experiment & Theory \\
\hline$A / \mathrm{MHz}$ & $9695.27280(60)$ & 9693.2567 & $9522.2481(12)$ & 9516.0036 & $9721.5291(17)$ & 9716.6844 \\
\hline$B / \mathrm{MHz}$ & $9188.15572(59)$ & 9191.9578 & 9353.9384(11) & 9362.2887 & $9135.7459(12)$ & 9142.5408 \\
\hline$C / \mathrm{MHz}$ & $4716.17271(55)$ & 4716.3799 & $4717.38619(35)$ & 4717.6589 & $4708.48056(66)$ & 4708.8368 \\
\hline$D_{J} / \mathrm{kHz}$ & $3.21549(45)$ & 3.1632 & $3.19784(45)$ & 3.1510 & $3.2036(11)$ & 3.1459 \\
\hline$D_{J K} / \mathrm{kHz}$ & $-5.06600(40)$ & -4.9784 & $-5.05961(57)$ & -4.9705 & $-5.0516(15)$ & -4.9506 \\
\hline$D_{K} / \mathrm{kHz}$ & $2.18898(44)$ & 2.1506 & $2.19760(40)$ & 2.1527 & $2.1840(12)$ & 2.1389 \\
\hline$d_{1} / \mathrm{kHz}$ & $0.066679(100)$ & 0.0657 & $-0.03881(25)$ & -0.0310 & $0.04675(65)$ & 0.0458 \\
\hline$d_{2} / \mathrm{kHz}$ & $0.020636(47)$ & 0.0209 & {$[-0.0033]$} & -0.0033 & $0.00511(54)$ & 0.0060 \\
\hline$H_{J} / \mathrm{mHz}$ & [1.3169] & 1.3169 & [1.3326] & 1.3326 & [1.3100] & 1.3100 \\
\hline$H_{J K} / \mathrm{mHz}$ & {$[-5.3526]$} & -5.3526 & {$[-5.3844]$} & -5.3844 & {$[-5.3218]$} & -5.3218 \\
\hline$H_{K J} / \mathrm{mHz}$ & [6.7607] & 6.7607 & [6.7795] & 6.7795 & [6.7177] & 6.7177 \\
\hline$H_{K} / \mathrm{mHz}$ & {$[-2.7233]$} & -2.7233 & {$[-2.7263]$} & -2.7263 & {$[-2.7045]$} & -2.7045 \\
\hline$h_{1} / \mathrm{mHz}$ & {$[0.0488]$} & 0.0488 & {$[0.0178]$} & 0.0178 & [0.0414] & 0.0414 \\
\hline$h_{2} / \mathrm{mHz}$ & {$[-0.0443]$} & -0.0443 & {$[-0.0267]$} & -0.0267 & {$[-0.0292]$} & -0.0292 \\
\hline$h_{3} / \mathrm{mHz}$ & {$[-0.0061]$} & -0.0061 & {$[-0.0103]$} & -0.0103 & {$[-0.0129]$} & -0.0129 \\
\hline$L_{J K K} / \mu \mathrm{Hz}$ & -1.98 & - & -1.98 & - & -1.98 & - \\
\hline$\chi_{c c}^{(1)} / \mathrm{MHz}$ & - & - & $-2.608(35)$ & -2.666 & $-2.592(24)$ & -2.666 \\
\hline$\chi_{b b}^{(1)}-\chi_{a a}^{(1)} / \mathrm{MHz}$ & - & - & {$[0]$} & -0.071 & {$[0]$} & -0.071 \\
\hline$\chi_{c c}^{(3)} / \mathrm{MHz}$ & $2.265(39)$ & 2.21 & $2.203(47)$ & 2.21 & - & - \\
\hline$\chi_{b b}^{(3)}-\chi_{a a}^{(3)} / \mathrm{MHz}$ & $4.812(56)$ & 5.74 & $5.720(68)$ & 5.74 & - & - \\
\hline No. of lines & 248 & - & 399 & - & 244 & - \\
\hline \multirow[t]{3}{*}{$\sigma / \mathrm{kHz}$} & 32.5 & - & 33.8 & - & 32.7 & - \\
\hline & \multicolumn{2}{|c|}{${ }^{13} \mathrm{C}(4)$} & \multicolumn{2}{|c|}{${ }^{13} \mathrm{C}(5)$} & & \\
\hline & Experiment & Theory & Experiment & Theory & & \\
\hline$A / \mathrm{MHz}$ & $9573.81156(53)$ & 9576.1789 & $9632.88401(69)$ & 9626.3298 & & \\
\hline$B / \mathrm{MHz}$ & $9285.89570(41)$ & 9286.6274 & $9223.79544(49)$ & 9232.0577 & & \\
\hline$C / \mathrm{MHz}$ & $4712.53945(32)$ & 4712.9821 & $4710.66720(35)$ & 4710.9362 & & \\
\hline$D_{J} / \mathrm{kHz}$ & $3.20500(31)$ & 3.1559 & $3.19777(34)$ & 3.1456 & & \\
\hline$D_{J K} / \mathrm{kHz}$ & $-5.06574(35)$ & -4.9750 & $-5.04990(41)$ & -4.9574 & & \\
\hline$D_{K} / \mathrm{kHz}$ & $2.19660(29)$ & 2.1524 & $2.18893(41)$ & 2.1454 & & \\
\hline$d_{1} / \mathrm{kHz}$ & $0.069913(77)$ & 0.0649 & $-0.00468(15)$ & -0.0031 & & \\
\hline$d_{2} / \mathrm{kHz}$ & $0.021625(40)$ & 0.0227 & $-0.009635(45)$ & -0.0078 & & \\
\hline$H_{J} / \mathrm{mHz}$ & {$[1.3210]$} & 1.3210 & [1.3259] & 1.3259 & & \\
\hline$H_{J K} / \mathrm{mHz}$ & {$[-5.3628]$} & -5.3628 & {$[-5.3645]$} & -5.3645 & & \\
\hline$H_{K J} / \mathrm{mHz}$ & [6.7713] & 6.7713 & [6.7576] & 6.7576 & & \\
\hline$H_{K} / \mathrm{mHz}$ & {$[-2.7278]$} & -2.7278 & {$[-2.7175]$} & -2.7175 & & \\
\hline$h_{1} / \mathrm{mHz}$ & {$[0.0414]$} & 0.0414 & {$[0.0341]$} & 0.0341 & & \\
\hline$h_{2} / \mathrm{mHz}$ & {$[-0.0284]$} & -0.0284 & {$[-0.0037]$} & -0.0037 & & \\
\hline$h_{3} / \mathrm{mHz}$ & {$[-0.0014]$} & -0.0014 & {$[-0.0051]$} & -0.0051 & & \\
\hline$L_{J K K} / \mu \mathrm{Hz}$ & -1.98 & - & -1.98 & - & & \\
\hline$\chi_{c c}^{(1)} / \mathrm{MHz}$ & $-2.566(21)$ & -2.666 & $-2.541(30)$ & -2.666 & & \\
\hline$\chi_{b b}^{(1)}-\chi_{a a}^{(1)} / \mathrm{MHz}$ & {$[0]$} & -0.071 & {$[0]$} & -0.071 & & \\
\hline$\chi_{c c}^{(3)} / \mathrm{MHz}$ & $2.281(27)$ & 2.21 & $2.171(29)$ & 2.21 & & \\
\hline$\chi_{b b}^{(3)}-\chi_{a a}^{(3)} / \mathrm{MHz}$ & $3.028(40)$ & 5.74 & {$[5.74]$} & 5.74 & & \\
\hline No. of lines & 422 & - & 360 & - & & \\
\hline$\sigma / \mathrm{kHz}$ & 25.9 & - & 27.6 & - & & \\
\hline
\end{tabular}




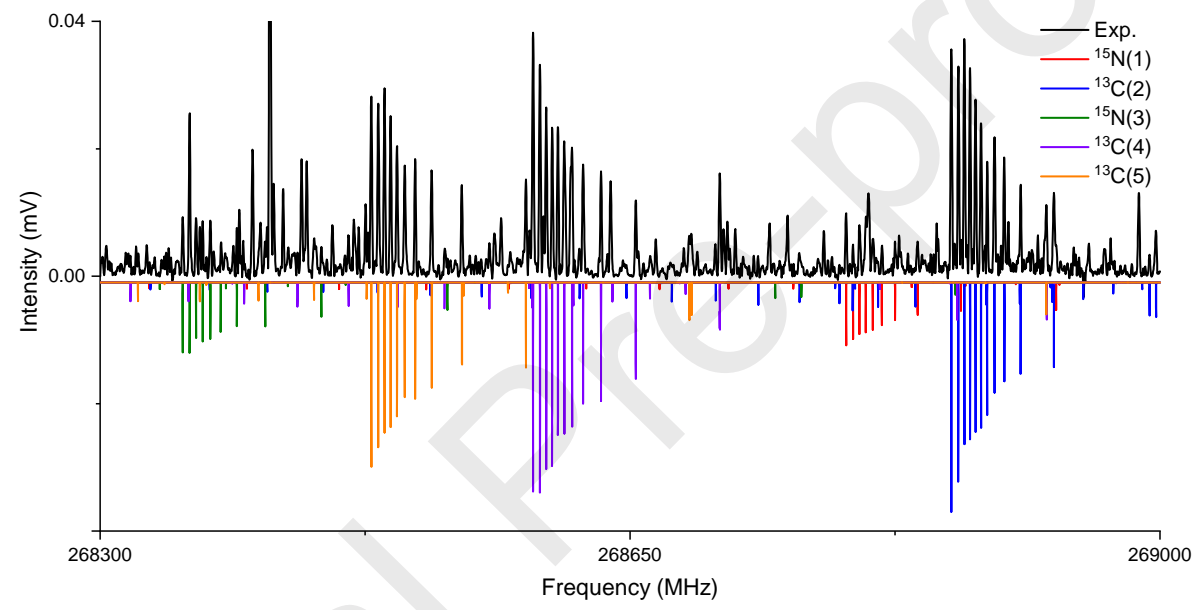

Figure 3: A zoom-in of the $260-295 \mathrm{GHz}$ region, showing the assignments of the singly-substituted isotopologues of imidazole, which do not exhibit hyperfine structure. The experimental spectra are shown in black, and the simulated spectra of the fitted rotational constants at $300 \mathrm{~K}$ are shown in colour. The atom labelling scheme is detailed in Table 1 and Figure 4. The simulated spectra intensities have been normalised to the experimental spectrum for clarity. 


\subsection{Molecular Structures}

The assigned isotopologues allowed us to determine the structure of imidazole in the gas phase. This was done to obtain both the substitution $\left(r_{s}\right)$ and the effective $\left(r_{0}\right)$ structures. These structures were determined by taking advantage of the presence of heavy-atom isotopologues in natural abundance, each of which has a distinct rotational spectrum. The $r_{s}$ structure analysis involves applying Kraitchman's equations to obtain the coordinates of the substituted heavy atom. The $r_{0}$ structure was determined by a least-squares fit, which calculated the bond lengths and angles that best reproduced the rotational constants of the main and heavy-atom isotopologues. Figure 4 shows an overlay of the theoretical structure optimised using the coupled-cluster composite method [5], shown in transparent colour, with the experimentally determined atom positions from the $r_{s}$ structure analysis, shown as solid spheres. The experimentally determined bond distances between the nuclei that make up the ring structure of imidazole and the experimentally determined internal angles of the five-membered ring are labelled. In Tables 4 and 5, these structural parameters are compared to the parameters presented in Refs. [5] and [19]. It can be seen that the substitution structure obtained from this analysis shows little deviation from that reported in Ref. [19], as can be expected from the good agreement of the rotational constants of each of the isotopologues. Indeed, whilst the experimental structural parameters - the bond lengths and internal bond angles - are of comparable quality to those in Ref. [19], the rotational constants are more precise by up to two significant figures / orders of magnitude. ${ }^{3}$ Our extended line lists and more precise rotational constants, which were derived from experimental data with an increased frequency range, are nevertheless important, especially for astrophysical applications at higher frequencies, with which our extended data sets overlap.

Table 4: A comparison of imidazole bond lengths, in $\AA$, determined in this work and by Christen $e t a l$. from the $r_{s}$ and $r_{0}$ structure analysis [19], and those from the structure optimised using the coupled-cluster composite method [5] and the MP2/aug-cc-pVTZ level of theory.

\begin{tabular}{lllllll}
\hline \multirow{2}{*}{ Bond length / $\AA$} & \multicolumn{2}{c}{$r_{s}$} & \multicolumn{2}{c}{ Theory } \\
& This work & $\begin{array}{l}r_{0} \\
\text { et al. }[19]\end{array}$ & This work & $\begin{array}{l}\text { Christen } \\
\text { et al. }[19]\end{array}$ & $\begin{array}{l}\text { Giuliano } \\
\text { et al. }[5]\end{array}$ & $\begin{array}{l}\text { This work } \\
\text { MP2 }\end{array}$ \\
\hline $\mathrm{N}(1)-\mathrm{C}(2)$ & $1.365(6)$ & 1.3643 & $1.367(6)$ & 1.3704 & 1.347 & 1.362 \\
$\mathrm{C}(2)-\mathrm{N}(3)$ & $1.321(7)$ & 1.3135 & $1.315(6)$ & 1.3164 & 1.286 & 1.322 \\
$\mathrm{~N}(3)-\mathrm{C}(4)$ & $1.373(7)$ & 1.3822 & $1.384(7)$ & 1.3875 & 1.370 & 1.373 \\
$\mathrm{C}(4)-\mathrm{C}(5)$ & $1.370(3)$ & 1.3638 & $1.370(6)$ & 1.3676 & 1.347 & 1.377 \\
$\mathrm{~N}(1)-\mathrm{C}(5)$ & $1.375(3)$ & 1.3774 & $1.383(7)$ & 1.3788 & 1.368 & 1.372 \\
\hline
\end{tabular}

This structure of imidazole can be compared directly to the structures of related molecules. Indeed, in Ref. [19], the imidazole structure was discussed in comparison with those of oxazole, pyrrole, and pyrazole. To add to these, and to put the structure of imidazole $\left(c\right.$-NHCHN $\left.\left(\mathrm{CH}_{2}\right)_{2}\right)$ in context with rings of the same elemental composition and connectivity, we present a comparison to the fully saturated ring analogues hydantoin $\left(c-\mathrm{NHC}(\mathrm{O}) \mathrm{NHCH}_{2} \mathrm{C}(\mathrm{O})\right)$ and imidazolidine $\left(c-\mathrm{NHCH}_{2} \mathrm{NH}\left(\mathrm{CH}_{2}\right)_{2}\right)$.

\footnotetext{
${ }^{3}$ The error in the structural parameters was not reported directly in Ref. [19], but we have reprocessed their data in order to calculate the associated errors in order to facilitate a thorough comparison. The relevant data can be found in the Supplementary Material.
} 
(a) $r_{s} / r_{0} /$ theory $(\AA)$

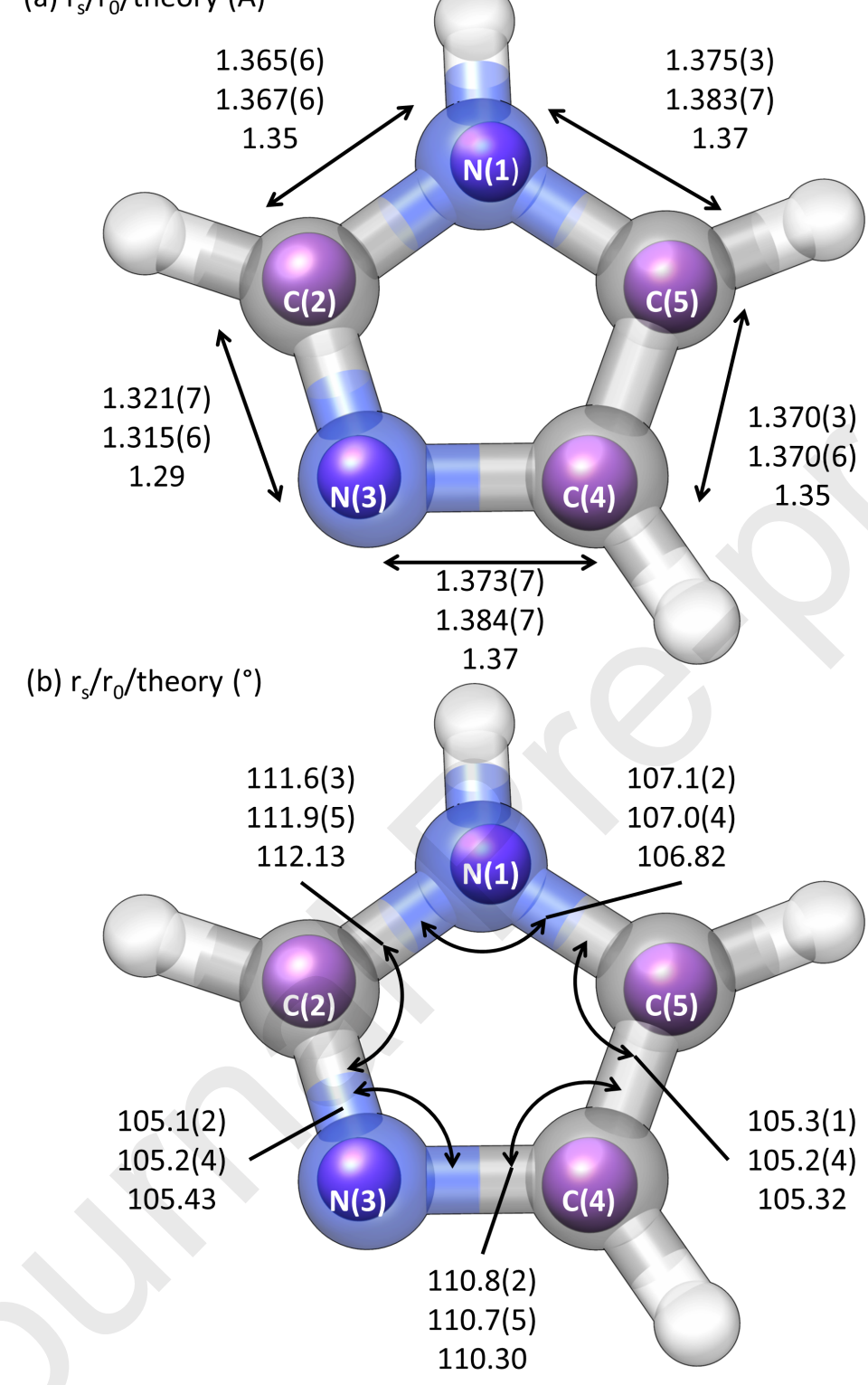

Figure 4: The theoretical structure of imidazole, optimised using the coupled-cluster composite method [5] and shown in transparent colour, is compared to the experimentally determined atom positions from the $r_{s}$ structure analysis, shown as solid spheres. Three sets of values are shown, which are from the $r_{s}$ structure analysis, the $r_{0}$ structure analysis, and from the coupled-cluster composite method. The bond lengths between the heavy atoms of the ring are labelled in (a), and the internal angles of the ring are given in (b). 
(a) Imidazole
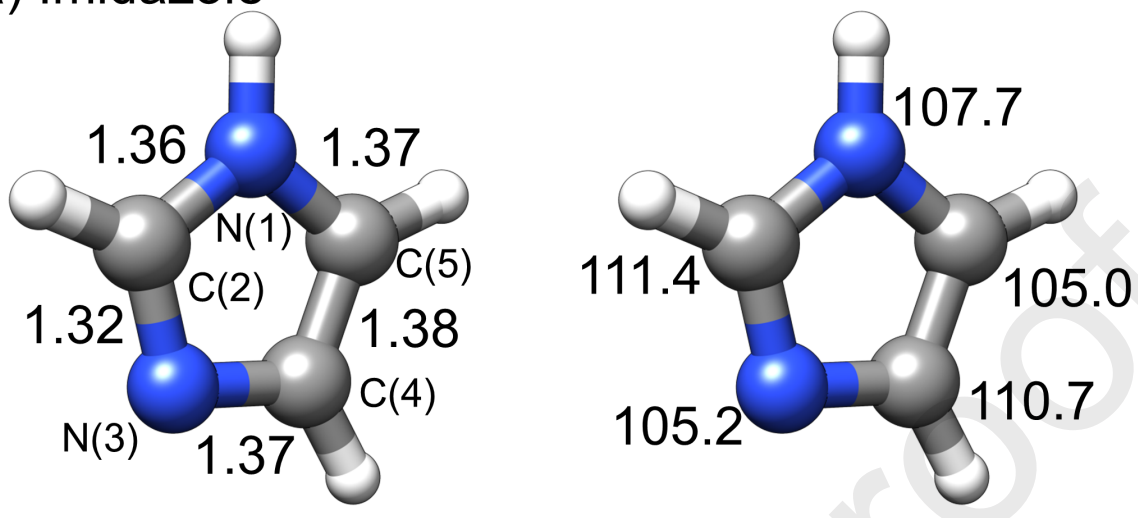

(b) Hydantoin
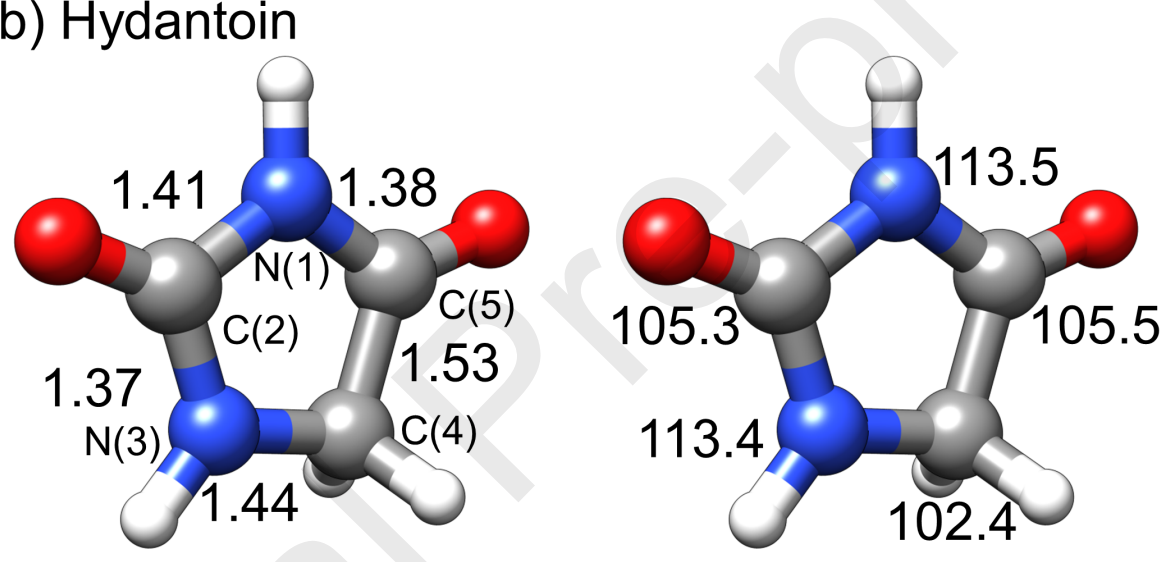

(c) Imidazolidine
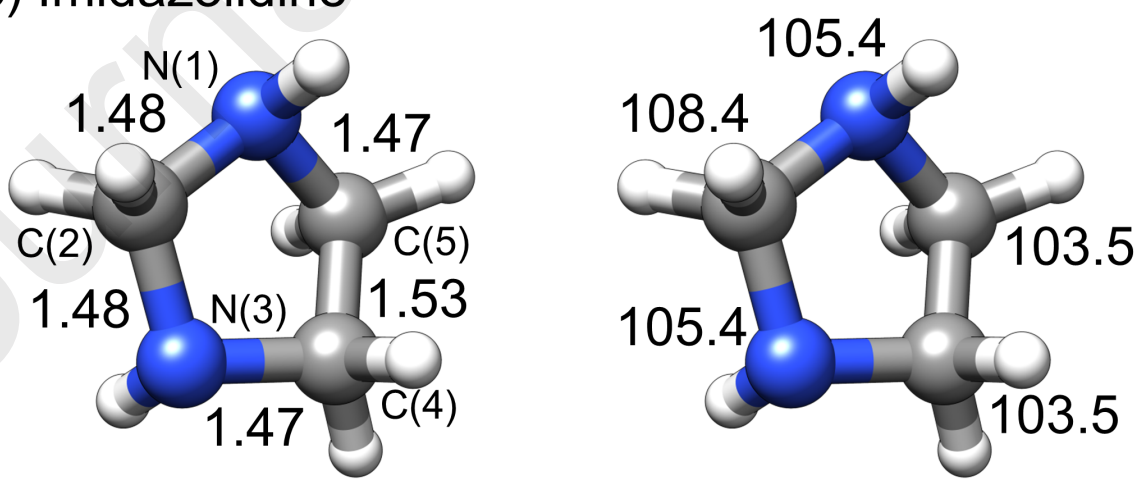

Figure 5: The structures of (a) imidazole, (b) hydantoin, and (c) imidazolidine. The bond lengths between the ring atoms are shown, in $\AA$, on the left side, while the internal angles are marked at the atom of the angle vertex, in degrees $\left({ }^{\circ}\right)$, on the right side. The values are all from structure optimisation calculations at the MP2/aug-cc-pVTZ level of theory. 
Table 5: A comparison of the internal bond angles of the imidazole ring, in degrees $\left(^{\circ}\right.$ ), determined in this work and by Christen et al. from the $r_{s}$ and $r_{0}$ structure analysis [19], and those from the structure optimised using the coupled-cluster composite method [5] and the MP2/aug-cc-pVTZ level of theory.

\begin{tabular}{lllllll}
\hline \multirow{2}{*}{ Bond angle $/^{\circ}$} & \multirow{2}{*}{${ }^{r_{s}}$ This work } & $\begin{array}{l}\text { Christen } \\
\text { et al. }[19]\end{array}$ & This work & $\begin{array}{l}r_{0} \text { Christen } \\
\text { et al. }[19]\end{array}$ & $\begin{array}{l}\text { Tiuliano } \\
\text { et al. }[5]\end{array}$ & $\begin{array}{l}\text { This work } \\
\text { MP2 }\end{array}$ \\
\hline $\mathrm{N}(1)-\mathrm{C}(2)-\mathrm{N}(3)$ & $111.6(3)$ & 111.99 & $111.9(5)$ & 111.85 & 112.1 & 111.4 \\
$\mathrm{C}(2)-\mathrm{N}(3)-\mathrm{C}(4)$ & $105.1(2)$ & 104.93 & $105.2(4)$ & 105.08 & 105.4 & 105.2 \\
$\mathrm{~N}(3)-\mathrm{C}(4)-\mathrm{C}(5)$ & $110.8(2)$ & 110.69 & $110.7(5)$ & 110.50 & 110.3 & 110.7 \\
$\mathrm{C}(4)-\mathrm{C}(5)-\mathrm{N}(1)$ & $105.3(1)$ & 105.48 & $105.2(4)$ & 105.65 & 105.3 & 105.0 \\
$\mathrm{C}(5)-\mathrm{N}(1)-\mathrm{C}(2)$ & $107.1(2)$ & 106.90 & $107.0(4)$ & 106.93 & 106.8 & 107.7 \\
\hline
\end{tabular}

Figure 5 shows the ring structures of the three molecules along with the relevant bond lengths and internal angles. The values are taken from structure optimisation calculations at the MP2/aug-cc-pVTZ level of theory, which were performed for this work, in particular because of the absence of the experimental gas-phase structure for imidazolidine in the literature. The main difference between the structures is the degree of saturation of the ring bonds, and this impacts the bond lengths. The $\mathrm{C}(2)-\mathrm{N}(3)$ bond illustrates this clearly. In the fully aromatic system, imidazole, where the bond is formally a carbon-nitrogen double bond, the experimental bond length is 1.321(7) $\AA$. This can be compared to the theoretical value for this bond length, which is $1.32 \AA$ at the MP2/aug-cc-pVTZ level of theory. This demonstrates the excellent agreement between the MP2 calculation and the $r_{s}$ structure (for most parameters, a better agreement than between the experimental structure and the structure from the coupled-cluster method), which we can exploit for further comparisons. The $\mathrm{C}(2)-\mathrm{N}(3)$ bond increases to 1.37 $\AA$ in hydantoin, in which the bond has some double bond character due to resonance effects. From an experimental point of view, as can be seen in Tables 6 and 7, the bond distance in hydantoin ${ }^{4}$ is $1.359(10) \AA$. This is also essentially the same as the theoretical value. The $\mathrm{C}(2)-\mathrm{N}(3)$ bond is the longest in the fully saturated imidazolidine, where it is a carbon-nitrogen single bond, at $1.48 \AA$. The $\mathrm{C}(5)-\mathrm{N}(1)$ bond in hydantoin also has some double bond character, and this bond, at $1.38 \AA$, is similar in length to the corresponding bond in imidazole ( $1.37 \AA$ ). The same bond in imidazolidine is longer, at $1.47 \AA$. The comparison of the experimental gas-phase structures shows a slight elongation in the hydantoin $\mathrm{C}(5)-\mathrm{N}(1)$ bond (1.401(5) $\AA$ ) when compared to the same imidazole bond $(1.375(3) \AA)$.

Another consequence of unsaturation and aromaticity is structure planarity. This can be characterised by the dihedral angles between the atoms in the ring, where $\Theta_{1}$ is the dihedral angle $\mathrm{N}(1)-\mathrm{C}(2)-\mathrm{N}(3)-\mathrm{C}(4), \Theta_{2}$ is $\mathrm{C}(2)-\mathrm{N}(3)-\mathrm{C}(4)-\mathrm{C}(5), \Theta_{3}$ is $\mathrm{N}(3)-\mathrm{C}(4)-$ $\mathrm{C}(5)-\mathrm{N}(1), \Theta_{4}$ is $\mathrm{C}(4)-\mathrm{C}(5)-\mathrm{N}(1)-\mathrm{C}(2)$, and $\Theta_{5}$ is $\mathrm{C}(5)-\mathrm{N}(1)-\mathrm{C}(2)-\mathrm{N}(3)$. In the fully saturated, non-aromatic imidazolidine, the ring structure is non-planar. The dihedral angles in imidazolidine are: $\Theta_{1}=11.1^{\circ}, \Theta_{2}=-27.8^{\circ}, \Theta_{3}=34.5^{\circ}, \Theta_{4}=-27.8^{\circ}$, and $\Theta_{5}=11.1^{\circ}$. These values are in contrast to those of the other two structures, where the dihedral angles are essentially zero for both imidazole and hydantoin. This planar structure is expected for imidazole because of its aromatic nature and for hydantoin

\footnotetext{
${ }^{4} \mathrm{~A}$ full account of the assigned transitions and the experimentally determined rotational constants and heavy-atom coordinates for hydantoin can be found in the Supplementary Material.
} 
Table 6: A comparison of hydantoin bond lengths, in $\AA$, determined from the $r_{s}$ structure analysis and the MP2/aug-cc-pVTZ structure optimisation calculation. The atom labelling scheme is detailed in Figure 5.

\begin{tabular}{lll}
\hline Bond length $/ \AA$ & $r_{s}$ & Theory \\
\hline $\mathrm{N}(1)-\mathrm{C}(2)$ & $1.389(6)$ & 1.407 \\
$\mathrm{C}(2)-\mathrm{N}(3)$ & $1.359(10)$ & 1.366 \\
$\mathrm{~N}(3)-\mathrm{C}(4)$ & $1.445(3)$ & 1.444 \\
$\mathrm{C}(4)-\mathrm{C}(5)$ & $1.516(9)$ & 1.526 \\
$\mathrm{~N}(1)-\mathrm{C}(5)$ & $1.401(5)$ & 1.374 \\
\hline
\end{tabular}

Table 7: A comparison of the internal bond angles of the hydantoin ring, in degrees $\left(^{\circ}\right.$ ), determined from the $r_{s}$ structure analysis and the MP2/aug-cc-pVTZ structure optimisation calculation. The atom labelling scheme is detailed in Figure 5.

\begin{tabular}{lll}
\hline Bond angles / & $r_{s}$ & Theory \\
\hline $\mathrm{N}(1)-\mathrm{C}(2)-\mathrm{N}(3)$ & $107.0(3)$ & 105.3 \\
$\mathrm{C}(2)-\mathrm{N}(3)-\mathrm{C}(4)$ & $112.7(2)$ & 113.4 \\
$\mathrm{~N}(3)-\mathrm{C}(4)-\mathrm{C}(5)$ & $102.7(2)$ & 102.4 \\
$\mathrm{C}(4)-\mathrm{C}(5)-\mathrm{N}(1)$ & $105.4(2)$ & 105.5 \\
$\mathrm{C}(5)-\mathrm{N}(1)-\mathrm{C}(2)$ & $112.2(5)$ & 113.5 \\
\hline
\end{tabular}

because of the carbonyl groups introducing resonance effects. The planarity can be explained by the hybridisation of the atoms in the ring motif. The $s p^{2}$-hybridised molecular orbitals in the imidazole ring locks the planar structure, whilst the $s p^{3}$-hybridised molecular orbitals in imidazolidine force some deviation from planarity.

The planarity of the imidazole ring can also be revealed by considering the second moments of inertia, $P_{i i}$, along the three axes, $i=a, b, c$. The values of $P_{i i}$ can indicate the extension of mass along the $i$ axis. The rotational constants, moments of inertia, and second moments of inertia for the imidazole normal isotopologue (reported in Ref. [5]) and the rare isotopologues (reported here) are detailed in Table 8, and the values were converted using the protocols contained in Ref. [49]. The tabulated values of $P_{c c}$, which were all calculated using the experimentally determined rotational constants, are around zero. Therefore, the mass of the imidazole ring atoms lie on the $a b$ plane; there is almost zero extension of mass along the $c$ axis. The fact that the $P_{c c}$ values for the isotopologues show little-to-no change when compared to the value for the normal isotopologue further shows that the substituted atoms lie on the $a b$ plane, reinforcing the conclusion of planarity.

Further structural information can be retrieved from the values of the nuclear quadrupole coupling constants, $\chi_{i}$, which are an indication of the electronic environment of the respective nuclei. In Ref. [50], the authors concluded that the hydantoin molecule contains two pyrrolic nitrogen nuclei. That is, the lone pairs of electrons on the nitrogen nuclei are within the aromatic ring system. The electrons are, therefore, unavailable for bonding to an external electrophile, as is the case in pyrrole. This was signified by the negative $\chi_{c c}$ values $-4.7338(70)$ and $-3.4635(75) \mathrm{MHz}$ for $\mathrm{N}(1)$ and $\mathrm{N}(3)$, respectively 
Table 8: The experimental rotational constants $(A, B, C)$, moments of inertia $\left(I_{a}, I_{b}\right.$, $\left.\mathrm{I}_{c}\right)$, and second moments of inertia $\left(\mathrm{P}_{a a}, \mathrm{P}_{b b}, \mathrm{P}_{c c}\right)$ for the normal isotopologue and heavy-atom isotopologues of imidazole.

\begin{tabular}{|c|c|c|c|}
\hline & Rotational constant $(\mathrm{MHz})$ & Moment of inertia $\left(\mathrm{amu} . \AA^{2}\right)$ & Second moment of inertia $\left(\mathrm{amu} . \AA^{2}\right)$ \\
\hline \multicolumn{4}{|c|}{ Main isotopologue } \\
\hline $\mathrm{A} / \mathrm{I}_{a} / \mathrm{P}_{a a}$ & 9725.30651 & 51.96535497 & 53.9272361 \\
\hline $\mathrm{B} / \mathrm{I}_{b} / \mathrm{P}_{b b}$ & 9373.99203 & 53.91289041 & 51.97970066 \\
\hline $\mathrm{C} / \mathrm{I}_{c} / \mathrm{P}_{c c}$ & 4771.91599 & 105.9069368 & -0.014345693 \\
\hline \multicolumn{4}{|c|}{${ }^{15} \mathrm{~N}(1)$} \\
\hline $\mathrm{A} / \mathrm{I}_{a} / \mathrm{P}_{a a}$ & 9695.27145 & 52.12633887 & 55.01783889 \\
\hline $\mathrm{B} / \mathrm{I}_{b} / \mathrm{P}_{b b}$ & 9188.15484 & 55.00331827 & 52.14085948 \\
\hline $\mathrm{C} / \mathrm{I}_{c} / \mathrm{P}_{c c}$ & 4716.17342 & 107.1586984 & -0.014520619 \\
\hline \multicolumn{4}{|c|}{${ }^{13} \mathrm{C}(2)$} \\
\hline $\mathrm{A} / \mathrm{I}_{a} / \mathrm{P}_{a a}$ & 9522.243 & 53.07352532 & 54.0430351 \\
\hline $\mathrm{B} / \mathrm{I}_{b} / \mathrm{P}_{b b}$ & 9353.9408 & 54.02845879 & 53.08810164 \\
\hline $\mathrm{C} / \mathrm{I}_{c} / \mathrm{P}_{c c}$ & 4717.38675 & 107.1311367 & -0.014576318 \\
\hline \multicolumn{4}{|c|}{${ }^{15} \mathrm{~N}(3)$} \\
\hline $\mathrm{A} / \mathrm{I}_{a} / \mathrm{P}_{a a}$ & 9721.5279 & 51.98555311 & 55.33353593 \\
\hline $\mathrm{B} / \mathrm{I}_{b} / \mathrm{P}_{b b}$ & 9135.7461 & 55.31885403 & 52.00023501 \\
\hline $\mathrm{C} / \mathrm{I}_{c} / \mathrm{P}_{c c}$ & 4708.48085 & 107.3337709 & -0.014681892 \\
\hline \multicolumn{4}{|c|}{${ }^{13} \mathrm{C}(4)$} \\
\hline $\mathrm{A} / \mathrm{I}_{a} / \mathrm{P}_{a a}$ & 9573.8098 & 52.78765879 & 54.43902448 \\
\hline $\mathrm{B} / \mathrm{I}_{b} / \mathrm{P}_{b b}$ & 9285.8951 & 54.42437154 & 52.80231172 \\
\hline $\mathrm{C} / \mathrm{I}_{c} / \mathrm{P}_{c c}$ & 4712.53924 & 107.2413362 & -0.014652938 \\
\hline \multicolumn{4}{|c|}{${ }^{13} \mathrm{C}(5)$} \\
\hline $\mathrm{A} / \mathrm{I}_{a} / \mathrm{P}_{a a}$ & 9632.88165 & 52.46394831 & 54.80539971 \\
\hline $\mathrm{B} / \mathrm{I}_{b} / \mathrm{P}_{b b}$ & 9223.79437 & 54.79079267 & 52.47855535 \\
\hline $\mathrm{C} / \mathrm{I}_{c} / \mathrm{P}_{c c}$ & 4710.66717 & 107.2839551 & -0.014607039 \\
\hline
\end{tabular}

[50]. For the vibronic ground state of imidazole, the $\chi_{c c}$ values indicate that the $\mathrm{N}(1)$ nucleus is pyrrolic and the $N(3)$ nucleus is pyridinic. In the latter case, the lone pair lies outside of the ring structure, and it is available for bonding to an external electrophile, as is the case in pyridine. The $\chi_{c c}$ values for the parent species of imidazole were determined to be $-2.5592(62)$ and 2.2293(66) MHz for $\mathrm{N}(1)$ and $\mathrm{N}(3)$, respectively [5]. This can also be seen in Table 3, where all of the values determined for $\chi_{c c}^{(1)}$ for the isotopologues are negative, and the $\chi_{c c}^{(3)}$ values are all positive.

The experimental analysis up to $295 \mathrm{GHz}$ for the vibrational states and the isotopologues allows for the prediction of the rotational spectra of these species to be accurate well beyond the laboratory frequency range. The most intense lines (to $\log _{10}($ Intensity)=$5.0 \mathrm{~nm}^{2} \mathrm{MHz}$ [34]) up to $600 \mathrm{GHz}$, for example, have frequency accuracies well below $1 \mathrm{MHz}$, meaning they can be relied upon for astrophysical searches. 


\section{Conclusions}

The broadband rotational spectrum of imidazole was recorded in the regions 2-8, 1215.5, 18-26, 75-110, and 260-295 GHz. The analysis of the parent species spectrum was previously reported [5], and the investigation is extended here to include three lowlying vibrationally excited states and the singly-substituted heavy-atom isotopologues.

The low-lying vibrational states assigned in the room-temperature data can be designated as an $\mathrm{N}-\mathrm{H}$ bond stretch (the fundamental mode $v_{21}$ ) and wagging motions along the C-C-N and N-C-N sections of the ring (the fundamental modes $v_{20}$ and $v_{19}$ ). Their relatively high energies reinforce the notion that the aromatic ring structure is rigid, especially when compared to the energies of the vibrationally excited states of the more flexible molecule imidazolidine. The experimental signal levels and predicted Boltzmann populations of the imidazole vibrational states compared to the ground state suggest searches for these species could be successful in warm regions of the ISM.

The gas-phase structure of imidazole was determined. When compared to the previous structure from Ref. [19], there was excellent agreement, with changes in the bond lengths (within $0.67 \%$ ) and bond angles (within $0.35 \%$ ) in the $r_{s}$ structures being negligible. The rotational spectrum of hydantoin was recorded in the $18-26 \mathrm{GHz}$ region, and an experimental gas-phase structure was also obtained for this heterocycle. The effect of the aromatic character of the imidazole ring was also explored, with comparisons being drawn to the experimental structure of hydantoin and the theoretical structure of the related molecule imidazolidine. Aromaticity explains the decrease in lengths of the ring bonds in imidazole when compared to the other cyclic molecules.

Searches for all of these species in the ISM are facilitated with the line lists, rotational constants, centrifugal distortion constants, and nuclear quadrupole coupling constants that result from our comprehensive analysis.

\section{Acknowledgements}

This work has been supported by the ERC Starting Grant ASTROROT (Grant Agreement Number 638027). B.E.A. acknowledges the International Max Planck Research School for Ultrafast Imaging and Structural Dynamics (IMPRS-UFAST). G.B. thanks Daniel Obenchain (now at Georg-August-Universitt Gttingen) for scientific discussions. A.P.C. gratefully acknowledges financial support by Universit Ca' Foscari Venezia (ADiR funds) and the facilities of the SCSCF ("Sistema per il Calcolo Scientifico di Ca' Foscari", a multiprocessor cluster system owned by Universita' Ca' Foscari Venezia). B. J. H. and B. H. P. are supported by the National Science Foundation. J.C.G. thanks the Centre National d'Etudes Spatiales (CNES) for financial support. 


\section{References}

[1] B. A. McGuire. 2018 Census of interstellar, circumstellar, extragalactic, protoplanetary disk, and exoplanetary molecules. Astrophysical Journal Supplement Series, 239(2):17, 2018.

[2] Z. Martins. The nitrogen heterocycle content of meteorites and their significance for the origin of life. Life, 8(3):28, 2018.

[3] W.M. Irvine, J. Ellder, A. Hjalmarson, E. Kollberg, O.E.H. Rydbeck, G.O. Sorensen, B. Bak, and H. Svanholt. Searches for interstellar imidazole and cyanoform. Astronomy $\mathcal{E}$ Astrophysics, 97:192-194, 1981.

[4] A. Belloche, H. S. P. Müller, R. T. Garrod, and K. M. Menten. Exploring molecular complexity with ALMA (EMoCA): Deuterated complex organic molecules in Sagittarius B2(N2). Astronomy E Astrophysics, 587:A91, 2016.

[5] B. M. Giuliano, L. Bizzocchi, A. Pietropolli Charmet, B. E. Arenas, A. L. Steber, M. Schnell, P. Caselli, B. J. Harris, B. H. Pate, J.-C. Guillemin, and A. Belloche. Rotational spectroscopy of imidazole: Improved rest frequencies for astrophysical searches. Astronomy E Astrophysics, 628:A53, 2019.

[6] B. A. McGuire, A. M. Burkhardt, S. Kalenskii, C. N. Shingledecker, A. J. Remijan, E. Herbst, and M. C. McCarthy. Detection of the aromatic molecule benzonitrile $\left(\mathrm{c}-\mathrm{C}_{6} \mathrm{H}_{5} \mathrm{CN}\right)$ in the interstellar medium. Science, 359(6372):202-205, 2018.

[7] M. C. McCarthy, K. L. K. Lee, R. A. Loomis, A. M. Burkhardt, C. N. Shingledecker, S. B. Charnley, M. A. Cordiner, E. Herbst, S. Kalenskii, E. R. Willis, C. Xue, A. J. Remijan, and B. A. McGuire. Interstellar detection of the highly polar five-membered ring cyanocyclopentadiene. Nature Astronomy, 2020.

[8] B. A. McGuire, R. A. Loomis, A. M. Burkhardt, and et al. Discovery of the interstellar polycyclic aromatic hydrocarbons 1- and 2-cyanonaphthalene. Science (Submitted), 2020.

[9] A. Belloche, H. S. P. Müller, K. M. Menten, P. Schilke, and C. Comito. Complex organic molecules in the interstellar medium: IRAM $30 \mathrm{~m}$ line survey of Sagittarius B2(N) and (M). Astronomy E Astrophysics, 559:A47, 2013.

[10] E. F. van Dishoeck. Laboratory astrophysics: Key to understanding the Universe. Proceedings of the International Astronomical Union, 15 (S350):3-14, 2019.

[11] V. Taquet, E. F. van Dishoeck, M. Swayne, D. Harsono, J. K. Jørgensen, L. Maud, N. F. W. Ligterink, H. S. P. Müller, C. Codella, K. Altwegg, A. Bieler, A. Coutens, M. N. Drozdovskaya, K. Furuya, M. V. Persson, M. L. R. van't Hoff, C. Walsh, and S. F. Wampfler. Linking interstellar and cometary $\mathrm{O}_{2}$ : A deep search for ${ }^{16} \mathrm{O}^{18} \mathrm{O}$ in the solar-type protostar IRAS 16293-2422. Astronomy $\mathcal{E}$ Astrophysics, 618:A11, 2018. 
[12] N. Brickhouse, G. J. Ferland, S. Milam, E. Sciamma-O’Brien, A. Smale, A. Spyrou, Phillip Stancil, L. Storrie-Lombardi, and G. M. Wahlgren. 2018 NASA Laboratory Astrophysics Workshop: Scientific Organizing Committee Report. Bulletin of the AAS, 52(2), 2020.

[13] R. A. Motiyenko, B. Tercero, J. Cernicharo, and L. Margulès. Rotational spectrum of formamide up to $1 \mathrm{THz}$ and first ISM detection of its vibrational state. Astronomy $\mathcal{E}$ Astrophysics, 548:A71, 2012.

[14] S.M. Fortman, I.R. Medvedev, C.F. Neese, and F.C. De Lucia. How complete are astrophysical catalogs for the millimeter and submillimeter spectral region? Astrophysical Journal Letters, 725:L11-L14, 2010.

[15] K. Furuya, Y. Aikawa, N. Sakai, and S. Yamamoto. Carbon isotope and isotopomer fractionation in cold dense cloud cores. The Astrophysical Journal, 731(1):38, 2011.

[16] J. Kraitchman. Determination of molecular structure from microwave spectroscopic data. American Journal of Physics, 21:17-24, 1953.

[17] G.L. Blackman, R.D. Brown, F.R. Burden, and I.R. Elsum. Nuclear quadrupole coupling in the microwave spectrum of imidazole. Journal of Molecular Spectroscopy, 60(1):63-70, 1976.

[18] J. H. Griffiths, A. Wardley, V.E. Williams, N.L. Owen, and J. Sheridan. Microwave spectra and structures of isothiazole, 1,2,4-oxadiazole and imidazole. Nature, 216:1301, 1967.

[19] D. Christen, J.H. Griffiths, and J. Sheridan. The microwave spectrum of imidazole; complete structure and the electron distribution from nuclear quadrupole coupling tensors and dipole moment orientation. Zeitschrift fr Naturforschung A, 36(12):1378-1385, 1982.

[20] M. Schwell, H. Jochims, H. Baumgärtel, and S. Leach. VUV photophysics and dissociative photoionization of pyrimidine, purine, imidazole and benzimidazole in the $718 \mathrm{eV}$ photon energy range. Chemical Physics, 353(1):145-162, 2008.

[21] Michael Frenklach and Eric D. Feigelson. Formation of polycyclic aromatic hydrocarbons in circumstellar envelopes. The Astrophysical Journal, 341:372, 1989.

[22] Alessandra Ricca, Charles W. Bauschlicher, and E.L.O. Bakes. A computational study of the mechanisms for the incorporation of a nitrogen atom into polycyclic aromatic hydrocarbons in the Titan haze. Icarus, 154(2):516-521, 2001.

[23] Ahmed M. Hamid, M. Samy El-Shall, Rifaat Hilal, Shaaban Elroby, and Saadullah G. Aziz. Unconventional hydrogen bonding to organic ions in the gas phase: Stepwise association of hydrogen cyanide with the pyridine and pyrimidine radical cations and protonated pyridine. The Journal of Chemical Physics, 141(5):054305, 2014. 
[24] V. P. Gupta, P. Tandon, P. Rawat, R. N. Singh, and A. Singh. Quantum chemical study of a new reaction pathway for the adenine formation in the interstellar space. Astronomy \& Astrophysics, 528:A129, 2011.

[25] D. Schmitz, V. A. Shubert, T. Betz, and M. Schnell. Multi-resonance effects within a single chirp in broadband rotational spectroscopy: The rapid adiabatic passage regime for benzonitrile. Journal of Molecular Spectroscopy, 280(1):7784, 2012.

[26] C. Pérez, A. Krin, A. L. Steber, J. C. López, Z. Kisiel, and M. Schnell. Wetting camphor: Multi-isotopic substitution identifies the complementary roles of hydrogen bonding and dispersive forces. The Journal of Physical Chemistry Letters, 7(1):154-160, 2016.

[27] M. Fatima, C. Prez, B. E. Arenas, M. Schnell, and A. L. Steber. Benchmarking a new segmented K-band chirped-pulse microwave spectrometer and its application to the conformationally rich amino alcohol isoleucinol. Physical Chemistry Chemical Physics, 22:17042-17051, 2020.

[28] J. L. Neill, B. J. Harris, A. L. Steber, K. O. Douglass, D. F. Plusquellic, and B. H. Pate. Segmented chirped-pulse Fourier transform submillimeter spectroscopy for broadband gas analysis. Optics Express, 21(17):19743, 2013.

[29] S. Gruet, C. Prez, A.L. Steber, and M. Schnell. Where's water? The many binding sites of hydantoin. Physical Chemistry Chemical Physics, 20:5545-5552, 2018.

[30] B. E. Arenas, S. Gruet, A. L. Steber, B. M. Giuliano, and M. Schnell. Chirpedpulse Fourier transform millimeter-wave spectroscopy of ten vibrationally excited states of i-propyl cyanide: Exploring the far-infrared region. Physical Chemistry Chemical Physics, 19(3):1751-1756, 2017.

[31] A. L. Steber, B. J. Harris, J. L. Neill, and B. H. Pate. An arbitrary waveform generator based chirped pulse Fourier transform spectrometer operating from 260 to $295 \mathrm{GHz}$. Journal of Molecular Spectroscopy, 280:3-10, 2012.

[32] B. J. Harris. A Chirped-Pulse Fourier Transform Millimeter Wave Spectrometer for Room Temperature, Gas Mixture Analysis. PhD thesis, The University of Virginia, 2014.

[33] C. M. Western. PGOPHER: A program for simulating rotational, vibrational and electronic spectra. Journal of Quantitative Spectroscopy and Radiative Transfer, 186:221-242, 2017.

[34] H. M. Pickett. The fitting and prediction of vibration-rotation spectra with spin interactions. Journal of Molecular Spectroscopy, 148(2):371-377, 1991.

[35] Z. Kisiel. PROSPE - Programs for Rotational Spectroscopy, 2015.

[36] Zbigniew Kisiel. Least-squares mass-dependence molecular structures for selected weakly bound intermolecular clusters. Journal of Molecular Spectroscopy, 218(1):58-67, 2003. 
[37] A. Pietropolli Charmet, P. Stoppa, N. Tasinato, and S. Giorgianni. Computing sextic centrifugal distortion constants by DFT: A benchmark analysis on halogenated compounds. Journal of Molecular Spectroscopy, 335:117 - 125, 2017.

[38] M. J. Frisch, G. W. Trucks, H. B. Schlegel, G. E. Scuseria, M. A. Robb, J. R. Cheeseman, G. Scalmani, V. Barone, G. A. Petersson, H. Nakatsuji, X. Li, M. Caricato, A. Marenich, J. Bloino, B. G. Janesko, R. Gomperts, B. Mennucci, H. P. Hratchian, J. V. Ortiz, A. F. Izmaylov, J. L. Sonnenberg, D. Williams-Young, F. Ding, F. Lipparini, F. Egidi, J. Goings, B. Peng, A. Petrone, D. Henderson, T. andRanasinghe, V. G. Zakrzewski, J. Gao, N. Rega, G. Zheng, W. Liang, M. Hada, M. Ehara, K. Toyota, R. Fukuda, J. Hasegawa, M. Ishida, T. Nakajima, Y. Honda, O. Kitao, H. Nakai, T. Vreven, K. Throssell, J. A. Montgomery, Jr., J. E. Peralta, F. Ogliaro, M. Bearpark, J. J. Heyd, E. Brothers, K. N. Kudin, V. N. Staroverov, T. Keith, R. Kobayashi, J. Normand, K. Raghavachari, A. Rendell, J. C. Burant, S. S. Iyengar, J. Tomasi, M. Cossi, J. M. Millam, M. Klene, C. Adamo, R. Cammi, J. W. Ochterski, R. L. Martin, K. Morokuma, O. Farkas, J. B. Foresman, and D. J. Fox. Gaussian 09, 2009.

[39] C. Perchard, A.-M. Bellocq, and A. Novak. Spectres de vibration de l'imidazole, de l'imidazole (D)-1, de l'imidazole (D3)-2,4,5 et de l'imidazole (D4) - Partie ii. Région entre 1700 et $30 \mathrm{~cm}^{-1}$. Journal de Chimie Physique et de Physico-Chimie Biologique, 62:1344-1358, 1965.

[40] S. Gruet, O. Pirali, M. Goubet, D. W. Tokaryk, and P. Brechignac. High-resolution far-infrared spectroscopy of N-substituted two-ring polycyclic aromatic hydrocarbons: An extended study. The Journal of Physical Chemistry A, 120(1):95-105, 2016.

[41] T.D Klots and W.B Collier. Heteroatom derivatives of indene Part 3. Vibrational spectra of benzoxazole, benzofuran, and indole. Spectrochimica Acta Part A: Molecular and Biomolecular Spectroscopy, 51(8):1291-1316, 1995.

[42] K. Vávra, K. Luková, P. Kania, J. Koucký, and Š. Urban. Rotational spectra of indole in the lowest vibrational states. Journal of Molecular Spectroscopy, 363:111175, 2019.

[43] Sieghard Albert, Karen Keppler Albert, Philippe Lerch, and Martin Quack. Synchrotron-based highest resolution Fourier transform infrared spectroscopy of naphthalene $\left(\mathrm{c}_{10} \mathrm{~h}_{8}\right)$ and indole $\left(\mathrm{c}_{8} \mathrm{~h}_{7} \mathrm{n}\right)$ and its application to astrophysical problems. Faraday Discussions, 150:71-99, 2011.

[44] H. Ozeki, R. Miyahara, H. Ihara, S. Todaka, K. Kobayashi, and M. Ohishi. Millimeter-wave spectroscopy of hydantoin, a possible precursor of glycine. Astronomy \& Astrophysics, 600:A44, 2017.

[45] Belloche, A., Garrod, R. T., Müller, H. S. P., Menten, K. M., Medvedev, I., Thomas, J., and Kisiel, Z. Re-exploring Molecular Complexity with ALMA (ReMoCA): interstellar detection of urea. Astronomy E Astrophysics, 628:A10, 2019. 
[46] W. Gordy and R. L. Cook. Microwave molecular spectra. New York: Wiley, 1984.

[47] L. Nygaard, R.L. Hansen, J.T. Noelsen, J. Rastrup-Andersen, O.O. Srensen, and P.A. Steiner. Microwave spectra of isotopic 1,3,4-oxadiazoles: Molecular structure, ${ }^{14} \mathrm{~N}$ quadrupole coupling constants, and centrifugal distortion constants of 1,3,4-oxadiazole. Journal of Molecular Structure, 12:59 - 69, 1972.

[48] F. Mata, M.J. Quintana, and G.O. Srensen. Microwave spectra of pyridine and monodeuterated pyridines. Revised molecular structure of pyridine. Journal of Molecular Structure, 42:1 - 5, 1977.

[49] R.K. Bohn, J.A. Montgomery, H.H. Michels, and J.A. Fournier. Second moments and rotational spectroscopy. Journal of Molecular Spectroscopy, 325:42 - 49, 2016.

[50] E. R. Alonso, L. Kolesnikov, and J. L. Alonso. Laser ablated hydantoin: A high resolution rotational study. The Journal of Chemical Physics, 147(12):124312, 2017. 